\title{
Gamificação: Um aplicativo para o ensino da Língua Brasileira de Sinais
}

\author{
Paul Symon Ribeiro Rocha1, Rommel Wladimir de Lima', Robson Locatelli \\ Macedo $^{1}$, Cicilia Raquel Maia Leite ${ }^{1}$, Francisco Milton Mendes Neto ${ }^{1}$ \\ ${ }^{1}$ Programa de Pós-Graduação em Ciência da Computação (PPgCC) \\ Universidade do Estado do Rio Grande do Norte (UERN) \\ Universidade Federal Rural do Semi-Árido (UFERSA) \\ Mossoró - RN - Brasil \\ \{paulsymon.ribeiro, ciciliamaia\}@gmail.com, \\ miltonmendes@ufersa.edu.br, rommelwladimir@uern.br, \\ robson_loc@hotmail.com
}

\begin{abstract}
This article aims to present an educational mobile application based on gamification techniques that aims to support the teaching-learning Brazilian Sign Language (LIBRAS). This technique is gaining visibility for their ability to create meaningful experiences when applied in contexts of everyday life. Therefore, this application is intended to make the user can learn LIBRAS in a fun, intuitive and motivating, facilitating their learning process. Thus, over time communication and interaction with the deaf community will no longer be a barrier.
\end{abstract}

Resumo. Este artigo tem como finalidade apresentar um aplicativo móvel educacional baseado em técnicas de gamificação que tem o objetivo de apoiar o ensino-aprendizagem da Língua Brasileira de Sinais (LIBRAS). Essa técnica vem ganhando visibilidade por sua capacidade de criar experiências significativas quando aplicada em contextos da vida cotidiana. Com isso, esse aplicativo pretende fazer com que o usuário possa aprender LIBRAS de uma forma divertida, intuitiva e motivadora, facilitando o seu processo de aprendizagem. Assim, com o passar do tempo a comunicação e interação com a comunidade surda deixará de ser uma barreira.

\section{Introdução}

Gamificação (do original em inglês gamification) corresponde ao uso de mecanismos de jogos que tem como objetivo resolver problemas práticos ou de despertar engajamento de um público específico. Nos últimos anos gamificação vem sido aplicada em diversos ambientes, tais como: no esporte, na saúde, no aumento de produtividade de empresas, na educação, entre outros ambientes. Atualmente existe um crescente interesse pela gamificação, suas aplicações e implicações [Vianna et al. 2013; Koster 2013].

O foco da gamificação é envolver emocionalmente o jogador utilizando mecanismos provenientes dos jogos, que proporciona a criação de um ambiente propício ao engajamento do indivíduo [Da Silva et al. 2014]. O termo "gamificação" ganhou 
V Congresso Brasileiro de Informática na Educação (CBIE 2016)

Anais dos Workshops do V Congresso Brasileiro de Informática na Educação (CBIE 2016)

popularidade a partir de uma apresentação de $\mathrm{TED}^{1}$ (Technology, Entertainment, Design) realizada por Jane McGonigal, famosa game designer norte americana e autora do livro "Reality Is Broken: Why Games Make us Better and How they Can Change the World".

Entender gamificação implica na compreensão do que são jogos. Um jogo é um sistema no qual jogadores se engajam em um desafio abstrato, definido por regras, interatividade e feedback, e que gera um resultado quantificável frequentemente gerando uma reação emocional [Kapp 2012]. É uma atividade voluntária feita espontaneamente pelo indivíduo, que proporciona prazer e permite o desenvolvimento de novas habilidades de pensamentos e cognição [Furió et al. 2013].

Acreditando na perspectiva de que jogos trazem resultados positivos aos jogadores, este presente trabalho apresenta um aplicativo móvel baseado em técnicas de gamificação com o objetivo de colaborar com o ensino e aprendizado da Língua Brasileira de Sinais.

Baseado no exposto, este trabalho está dividido da seguinte forma: seção 2 descreve brevemente alguns campos de aplicação da técnica de gamificação. Em seguida a seção 3 descreve o aplicativo desenvolvido. A seção 4 descreve os resultados esperados com o uso do aplicativo. Por fim, a seção 5 descreve as considerações finais e trabalhos futuros.

\section{Campos de aplicação da técnica de gamificação}

Gamificação é uma ferramenta multidisciplinar que abrange uma gama de domínios, teorias de pensamentos, metodologias, e várias razões para sua implementação. Seres humanos sentem-se fortemente atraídos por jogos.

A seguir, serão apresentados alguns exemplos, provenientes de campos diversos, que servirão para dimensionar os potenciais benefícios que podem ser alcançados por meio da gamificação. Neste artigo serão abordados dois campos: educação e saúde.

\subsection{Gamificação na educação}

A gamificação na educação tem a capacidade de tornar as aulas e disciplinas mais atraentes, produtivas e mais eficientes para os alunos e professores. De acordo com [Borges et al. 2014] a gamificação aplicada à educação tem como objetivos principais: aprimorar determinadas habilidades; apresentar desafios que dão contexto a aprendizagem; engajar os alunos em atividades mais participativas; potencializar o aprendizado de um determinado conteúdo; favorecer a mudança de comportamento e proporcionar mecanismos de socialização e aprendizagem em grupo.

A gamificação na educação faz com que o jogador se divirta em um jogo ao mesmo tempo que vai aprendendo conceitos novos. A cada acerto, ganha premiações e o direito de passar para o próximo nível, e a cada novo nível, novos conceitos são apresentados. Essa técnica aparece como uma nova possibilidade de conectar o ambiente escolar ao universo dos jovens. Ao invés do sistema tradicional, elementos de

\footnotetext{
${ }^{1}$ TED é uma organização sem fins lucrativos dedicada às "Ideias que merecem ser compartilhadas" que reúne inúmeras palestras com conteúdo de qualidade.
} 
jogos na educação promovem experiências que envolvem emocionalmente e cognitivamente os alunos [Da Silva et al. 2014].

Uma das ferramentas para o ensino de idiomas que utiliza conceitos de gamificação mais difundidas do mundo atualmente é o aplicativo Duolingo ${ }^{2}$. Focado em vocabulário, escrita e compreensão oral, o aprendizado é absolutamente customizado de acordo a experiência do usuário [Da Silva et al. 2014; Vianna et al. 2013].

\subsection{Gamificação na saúde}

Gamificação na saúde é utilizada para diversos fins, tais como: para treinamento médico, educação de saúde, terapia psicológica, reabilitação física ou cognitiva, participações mais ativas de um paciente a um determinado tratamento, entre outros.

Jogos tem a característica de desviar totalmente a atenção do paciente, portanto eles também podem ser utilizados no tratamento da dor. Eles oferecem desafios, requerem habilidades e são incrivelmente motivadores, e é de motivação que é preciso para auxiliar os pacientes em processos de reabilitação [Rego, Moreira e Reis 2010].

Como exemplo de uma ferramenta utilizada na área da saúde, a ferramenta SuperBetter ${ }^{3}$, desenvolvida por Jane McGonigal, auxilia pacientes a conquistarem progressos relacionados a melhoria de seus estados clínicos. As pessoas ao redor do mundo estão usando SuperBetter para vencer a depressão, superar a ansiedade, lidar com doença crônica ou dor crônica, recuperação de stress pós-traumático, adotar um novo hábito, entre outros benefícios. Dessa forma, a batalha pela cura de uma doença séria pode transformar-se em um desafio prazeroso, onde o maior prêmio será $\mathrm{o}$ restabelecimento da própria qualidade de vida [Vianna et al. 2013].

\section{O jogo e o aprendizado de LIBRAS}

O aplicativo aqui proposto trata-se de um jogo com potencial educativo para auxiliar no processo de ensino-aprendizado de LIBRAS. Na sua primeira versão o aplicativo denominado "Serious LIBRAS" conta com o nível iniciante, nesse mesmo nível existem dois subníveis (fases 1 e 2). Os níveis intermediário e avançado serão implementados em versões posteriores. A tela inicial do aplicativo pode ser visualizado na Figura 1 ao lado esquerdo da imagem.

Ao clicar no botão do nível iniciante, um alerta é emitido com a seguinte mensagem: "Deseja aprender o alfabeto LIBRAS antes de responder as questões?", como pode ser observado na Figura 1 ao centro da imagem. Caso o usuário deseje aprender ou revisar o alfabeto, posteriormente será direcionado para a tela de seleção das fases, a Figura 1 ao lado direito ilustra a interface dessa atividade.

A fase 1 do nível iniciante corresponde a verificação de aprendizado das letras do alfabeto em LIBRAS, como pode ser observado na Figura 2 ao lado esquerdo da imagem. A imagem correspondente ao sinal da letra em LIBRAS é exibida na parte superior, logo abaixo são dadas quatro opções de respostas.

\footnotetext{
${ }^{2}$ Site: www.duolingo.com.

${ }^{3}$ Site: www.superbetter.com.
} 
V Congresso Brasileiro de Informática na Educação (CBIE 2016)

Anais dos Workshops do V Congresso Brasileiro de Informática na Educação (CBIE 2016)

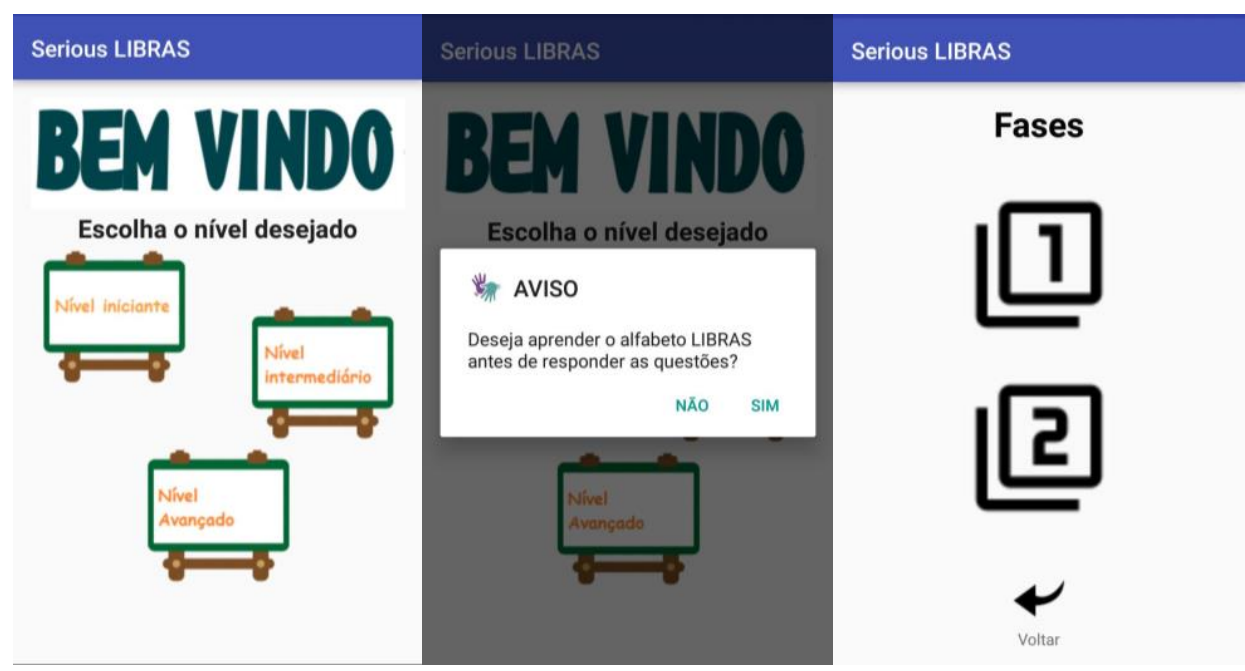

Figura 1.Interfaces do aplicativo "Serious LIBRAS".

Ao selecionar uma das opções e clicar no botão "Enviar resposta", uma mensagem é exibida em seguida informando o acerto ou erro da questão. Ao final do questionário, a quantidade de acertos é verificada e um alerta educativo é exibido, conforme Figura 2 ao centro da imagem, esse alerta contém a margem de acerto do usuário.

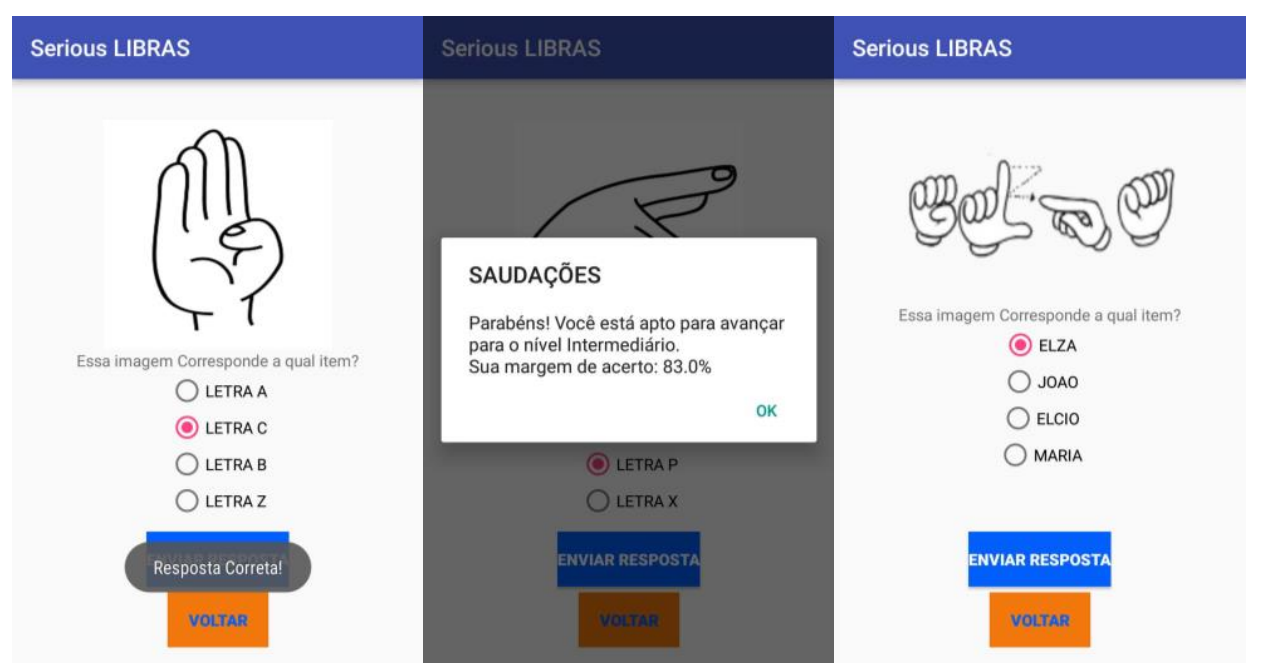

Figura 2. Interfaces do aplicativo "Serious LIBRAS".

A fase 2 corresponde a verificação de aprendizagem sobre palavras em LIBRAS, a Figura 2 a direita da imagem ilustra a interface dessa fase. Os princípios são os mesmos da fase 1 .

A versão atual do aplicativo foi desenvolvida para ser utilizada em dispositivos que possuem o sistema operacional Android. Além disso o usuário deve ter o domínio de leitura para poder utilizar o aplicativo de forma eficiente.

\section{Resultados esperados}

Espera-se que nessa versão atual do aplicativo, os usuários consigam aprender a datilologia. Datilologia é na verdade o alfabeto manual em LIBRAS, que segundo [Fernandes e Romeiro 2016, p178-179] 
V Congresso Brasileiro de Informática na Educação (CBIE 2016)

Anais dos Workshops do V Congresso Brasileiro de Informática na Educação (CBIE 2016)

[...] é comumente usada pela maioridade dos surdos, como, nas expressões linguísticas quando não há um sinal especifico, para nomes próprios, estrangeiros, e até como o meio de relacionar com o ouvinte, quando este não entende a Libras.

A datilologia é de grande ajuda aos ouvintes que desejam manter um contato mais próximo com os surdos, já que é um sistema que apresenta o alfabeto das línguas orais escritas fazendo o uso das mãos.

\section{Considerações finais e trabalhos futuros}

A gamificação se mostra como uma grande ferramenta para se explorar novos territórios e dar novos significados aos velhos processos, transformando-os em novas experiências, relevantes, ricas, capazes de gerar um enorme engajamento e uma surpreendente motivação entre os indivíduos. O aplicativo "Serious LIBRAS" foi desenvolvido para facilitar o aprendizado de LIBRAS por meio de elementos de jogos. É importante que o jogador perceba a transição entre o básico, o intermediário e o avançado para que ele saiba da sua evolução de aprendizagem e seja instigado a aprender cada vez mais. Em versões futuras, os níveis intermediário e avançado serão desenvolvidos, com o objetivo de proporcionar o ensino de mais conteúdos sobre LIBRAS.

\section{Agradecimentos}

Os autores agradecem a agencia brasileira de pesquisa CAPES, a Universidade Federal Rural do Semiárido (UFERSA) e a Universidade do Estado do Rio Grande do Norte (UERN) pelo apoio financeiro e infraestrutura disponibilizada.

\section{Referências}

Borges, S. de S. et al. (2014) "A systematic mapping on gamification applied to education", In: Proceedings of the 29th Annual ACM Symposium on Applied Computing. ACM, 2014. p. 216-222.

Da Silva, A. R. L. et al. (2014) "Gamificação na Educação”. Pimenta Cultural.

Fernandes, C. C.; Romeiro, C. de A.. (2016) "A contribuição da datilologia como estratégia metodológica no processo de alfabetização". Revista diálogos, v. 4, n. 1, p. 170-185, 2016.

Furió, D. et al. (2013) "The effects of the size and weight of a mobile device on an educational game”. Computers \& Education, v. 64, p. 24-41.

Kapp, K. M. (2012) "The gamification of learning and instruction: game-based methods and strategies for training and education". John Wiley \& Sons.

Koster, R. (2013) Theory of fun for game design. " O'Reilly Media, Inc.".

Rego, P.; Moreira, P. M.; Reis, L. P. (2010) "Serious games for rehabilitation: A survey and a classification towards a taxonomy". In: Information Systems and Technologies (CISTI), 5th Iberian Conference on. IEEE. p. 1-6.

Vianna, Y. et al. (2013) Gamification Inc.: como reinventar empresas a partir de jogos. 